\title{
Strong convergence of the hybrid method for a finite family of nonspreading mappings and variational inequality problems
}

\section{Atid Kangtunyakarn*}

"Correspondence:

beawrock@hotmail.com Department of Mathematics,

Faculty of Science, King Mongkut's Institute of Technology Ladkrabang,

Bangkok 10520, Thailand

\begin{abstract}
In this paper, we prove a strong convergence theorem by the hybrid method for finding a common element of the set of fixed points of a finite family of nonspreading mappings and the set of solutions of a finite family of variational inequality problems.
\end{abstract}

Keywords: nonspreading mapping; quasi-nonexpansive mapping; S-mapping

\section{Introduction}

Let $C$ be a nonempty closed convex subset of a real Hilbert space $H$. Then a mapping $T: C \rightarrow C$ is said to be nonexpansive if $\|T x-T y\| \leq\|x-y\|$ for all $x, y \in C$. Recall that the mapping $T: C \rightarrow C$ is said to be quasi-nonexpansive if $\|T x-p\| \leq\|x-p\|, \forall x \in C$ and $\forall p \in$ $F(T)$, where $F(T)$ denotes the set of fixed points of $T$. In 2008, Kohsaka and Takahashi [1] introduced the mapping $T$ called the nonspreading mapping in Hilbert spaces $H$ and defined it as follows: $2\|T x-T y\|^{2} \leq\|T x-y\|^{2}+\|x-T y\|^{2}, \forall x, y \in C$.

Let $A: C \rightarrow H$. The variational inequality problem is to find a point $u \in C$ such that

$$
\langle A u, v-u\rangle \geq 0
$$

for all $v \in C$. The set of solutions of (1.1) is denoted by $\operatorname{VI}(C, A)$.

The variational inequality has emerged as a fascinating and interesting branch of mathematical and engineering sciences with a wide range of applications in industry, finance, economics, social, ecology, regional, pure and applied sciences; see, e.g., [2-5].

A mapping $A$ of $C$ into $H$ is called inverse-strongly monotone (see [6]) if there exists a positive real number $\alpha$ such that

$$
\langle x-y, A x-A y\rangle \geq \alpha\|A x-A y\|^{2}
$$

for all $x, y \in C$. Throughout this paper, we will use the following notation:

1. $\rightarrow$ for weak convergence and $\rightarrow$ for strong convergence.

2. $\omega\left(x_{n}\right)=\left\{x: \exists x_{n_{i}} \rightarrow x\right\}$ denotes the weak $\omega$-limit set of $\left\{x_{n}\right\}$.

In 2008, Takahashi, Takeuchi and Kubota [7] proved the following strong convergence theorems by using the hybrid method for nonexpansive mappings in Hilbert spaces.

() 2012 Kangtunyakarn; licensee Springer. This is an Open Access article distributed under the terms of the Creative Commons Attribution License (http://creativecommons.org/licenses/by/2.0), which permits unrestricted use, distribution, and reproduction in any medium, provided the original work is properly cited. 
Theorem 1.1 Let $H$ be a Hilbert space and $C$ be a nonempty closed convex subset of $H$. Let $T$ be a nonexpansive mapping of $C$ into $H$ such that $F(T) \neq \emptyset$ and let $x_{0} \in H$. For $C_{1}=C$ and $u_{1} \in P_{C_{1}} x_{0}$, define a sequence $\left\{u_{n}\right\}$ of $C$ as follows:

$$
\left\{\begin{array}{l}
y_{n}=\alpha_{n} u_{n}+\left(1-\alpha_{n}\right) u_{n}, \\
C_{n+1}=\left\{z \in C_{n}:\left\|y_{n}-z\right\| \leq\left\|u_{n}-z\right\|\right\}, \\
u_{n+1}=P_{C_{n+1}} x_{0}, \quad n \in \mathbb{N},
\end{array}\right.
$$

where $0 \leq \alpha_{n} \leq a<1$ for all $n \in \mathbb{N}$. Then $\left\{u_{n}\right\}$ converges strongly to $z_{0}=P_{F(T)} x_{0}$.

In 2009, Iemoto and Takahashi [8] proved the convergence theorem of nonexpansive and nonspreading mappings as follows.

Theorem 1.2 Let $H$ be a Hilbert space, and let $C$ be a nonempty closed convex subset of $H$. Let $S$ be a nonspreading mapping of $C$ into itself, and let $T$ be a nonexpansive mapping of $C$ into itself such that $F(S) \cap F(T) \neq \emptyset$. Define a sequence $\left\{x_{n}\right\}$ as follows.

$$
\left\{\begin{array}{l}
x_{1} \in C, \\
x_{n+1}=\alpha_{n} x_{n}+\left(1-\alpha_{n}\right)\left(\beta_{n} S x_{n}+\left(1-\beta_{n}\right) T x_{n}\right)
\end{array}\right.
$$

for all $n \in \mathbb{N}$, where $\left\{\alpha_{n}\right\},\left\{\beta_{n}\right\} \subset[0,1]$. Then the following hold:

(i) If $\liminf _{n \rightarrow \infty} \alpha_{n}\left(1-\alpha_{n}\right)>0$ and $\sum_{n=1}^{\infty}\left(1-\beta_{n}\right)<\infty$, then $\left\{x_{n}\right\}$ converges weakly to $v \in F(S)$.

(ii) If $\sum_{n=1}^{\infty} \alpha_{n}\left(1-\alpha_{n}\right)=\infty$ and $\sum_{n=1}^{\infty} \beta_{n}<\infty$, then $\left\{x_{n}\right\}$ converges weakly to $v \in F(T)$.

(iii) If $\liminf _{n \rightarrow \infty} \alpha_{n}\left(1-\alpha_{n}\right)>0$ and $\liminf _{n \rightarrow \infty} \beta_{n}\left(1-\beta_{n}\right)>0$, then $\left\{x_{n}\right\}$ converges weakly to $v \in F(S) \cap F(T)$.

Inspired and motivated by these facts and the research in this direction, we prove the strong convergence theorem by the hybrid method for finding a common element of the set of fixed points of a finite family of nonspreading mappings and the set of solutions of a finite family of variational inequality problems.

\section{Preliminaries}

In this section, we collect and give some useful lemmas that will be used for our main result in the next section.

Let $C$ be a closed convex subset of a real Hilbert space $H$, let $P_{C}$ be the metric projection of $H$ onto $C$, i.e., for $x \in H, P_{C} x$ satisfies the property

$$
\left\|x-P_{C} x\right\|=\min _{y \in C}\|x-y\| .
$$

The following characterizes the projection $P_{C}$.

Lemma 2.1 (See [9]) Given $x \in H$ and $y \in C$. Then $P_{C} x=y$ if and only if the following inequality holds:

$$
\langle x-y, y-z\rangle \geq 0 \quad \forall z \in C .
$$


Lemma 2.2 (See [8]) Let $C$ be a nonempty closed convex subset of $H$. Then a mapping $S: C \rightarrow C$ is nonspreading if and only if

$$
\|S x-S y\|^{2} \leq\|x-y\|^{2}+2\langle x-S x, y-S y\rangle
$$

for all $x, y \in C$.

Example 2.3 Let $\mathcal{R}$ denote the reals with the usual norm. Let $T: \mathcal{R} \rightarrow \mathcal{R}$ be defined by

$$
T x=\left\{\begin{array}{l}
x-1 \quad \text { if } x \in(-\infty, 0], \\
-(x+1) \quad \text { if } x \in(0, \infty)
\end{array}\right.
$$

for all $x \in \mathcal{R}$.

To see that $T$ is a nonspreading mapping, if $x, y \in(0, \infty)$, then we have $T x=-(x+1)$ and $T y=-(y+1)$. From the definition of the mapping $T$, we have

$$
\begin{aligned}
|T x-T y|^{2} & =|-(x+1)-(-(y+1))|^{2} \\
& =|y-x|^{2}=|x-y|^{2}
\end{aligned}
$$

and

$$
\begin{aligned}
2\langle x-T x, y-T y\rangle & =2\langle x+x+1, y+y+1\rangle \\
& =2\langle 2 x+1,2 y+1\rangle \\
& =2(2 x+1)(2 y+1)>0 \quad(\text { since } x, y>0) .
\end{aligned}
$$

The above implies that

$$
|T x-T y|^{2}=|x-y|^{2}<|x-y|^{2}+2\langle x-T x, y-T y\rangle .
$$

For every $x, y \in(-\infty, 0]$, we have $T x=x-1$ and $T y=y-1$. From the definition of $T$, we have

$$
\begin{aligned}
|T x-T y|^{2} & =|x-1-(y-1)|^{2} \\
& =|x-y|^{2},
\end{aligned}
$$

and

$$
2\langle x-T x, y-T y\rangle=2\langle x-(x-1), y-(y-1)\rangle=2 .
$$

From above, we have

$$
|T x-T y|^{2}=|x-y|^{2}<|x-y|^{2}+2\langle x-T x, y-T y\rangle .
$$


Finally, for every $x \in(-\infty, 0]$ and $y \in(0, \infty)$, we have $T x=x-1$ and $T y=-(y+1)$. From the definition of $T$, we have

$$
\begin{aligned}
|T x-T y|^{2} & =|x-1+y+1|^{2}=|x+y|^{2}, \\
|x-y|^{2} & =x^{2}-2 x y+y^{2} \\
& =x^{2}+2 x y+y^{2}-4 x y \\
& \geq x^{2}+2 x y+y^{2} \quad(\text { since }-4 x y \geq 0) \\
& =(x+y)^{2}
\end{aligned}
$$

and

$$
\begin{aligned}
2\langle x-T x, y-T y\rangle & =2\langle x-(x-1), y+(y+1)\rangle \\
& =2\langle 1,2 y+1\rangle \\
& =2(2 y+1)>0 \quad(\text { since } y>0) .
\end{aligned}
$$

From above, we have

$$
\begin{aligned}
|T x-T y|^{2} & =|x+y|^{2}=(x+y)^{2} \\
& \leq|x-y|^{2} \\
& <|x-y|^{2}+2\langle x-T x, y-T y\rangle .
\end{aligned}
$$

Hence, for all $x, y \in \mathcal{R}$, we have

$$
|T x-T y|^{2}<|x-y|^{2}+2\langle x-T x, y-T y\rangle .
$$

Then $T$ is a nonspreading mapping.

Lemma 2.4 (See [1]) Let $H$ be a Hilbert space, let $C$ be a nonempty closed convex subset of $H$, and let $S$ be a nonspreading mapping of $C$ into itself. Then $F(S)$ is closed and convex.

Lemma 2.5 (See [9]) Let $H$ be a Hilbert space, let $C$ be a nonempty closed convex subset of $H$, and let $A$ be a mapping of $C$ into $H$. Let $u \in C$. Then for $\lambda>0$,

$$
u=P_{C}(I-\lambda A) u \quad \Leftrightarrow \quad u \in V I(C, A),
$$

where $P_{C}$ is the metric projection of $H$ onto $C$.

Lemma 2.6 (See [10]) Let C be a closed convex subset of a strictly convex Banach space E. Let $\left\{T_{n}: n \in \mathbb{N}\right\}$ be a sequence of nonexpansive mappings on C. Suppose $\bigcap_{n=1}^{\infty} F\left(T_{n}\right)$ is nonempty. Let $\left\{\lambda_{n}\right\}$ be a sequence of positive numbers with $\sum_{n=1}^{\infty} \lambda_{n}=1$. Then a mapping $S$ on $C$ defined by

$$
S(x)=\sum_{n=1}^{\infty} \lambda_{n} T_{n} x
$$

for $x \in C$ is well defined, nonexpansive and $F(S)=\bigcap_{n=1}^{\infty} F\left(T_{n}\right)$ holds. 
Lemma 2.7 (See [11]) Let E be a uniformly convex Banach space, $C$ be a nonempty closed convex subset of $E$, and $S: C \rightarrow C$ be a nonexpansive mapping. Then $I-S$ is demi-closed at zero.

Lemma 2.8 (See [12]) Let $C$ be a closed convex subset of $H$. Let $\left\{x_{n}\right\}$ be a sequence in $H$ and $u \in H$. Let $q=P_{C} u$. If $\left\{x_{n}\right\}$ is such that $\omega\left(x_{n}\right) \subset C$ and satisfies the condition

$$
\left\|x_{n}-u\right\| \leq\|u-q\|, \quad \forall n \in \mathbb{N},
$$

then $x_{n} \rightarrow q$, as $n \rightarrow \infty$.

In 2009, Kangtunyakarn and Suantai [13] introduced an $S$-mapping generated by $T_{1}, \ldots, T_{N}$ and $\lambda_{1}, \ldots, \lambda_{N}$ as follows.

Definition 2.1 Let $C$ be a nonempty convex subset of a real Banach space. Let $\left\{T_{i}\right\}_{i=1}^{N}$ be a finite family of (nonexpansive) mappings of $C$ into itself. For each $j=1,2, \ldots, N$, let $\alpha_{j}=$ $\left(\alpha_{1}^{j}, \alpha_{2}^{j}, \alpha_{3}^{j}\right) \in I \times I \times I$, where $I \in[0,1]$ and $\alpha_{1}^{j}+\alpha_{2}^{j}+\alpha_{3}^{j}=1$. Define the mapping $S: C \rightarrow C$ as follows:

$$
\begin{aligned}
& U_{0}=I \text {, } \\
& U_{1}=\alpha_{1}^{1} T_{1} U_{0}+\alpha_{2}^{1} U_{0}+\alpha_{3}^{1} I, \\
& U_{2}=\alpha_{1}^{2} T_{2} U_{1}+\alpha_{2}^{2} U_{1}+\alpha_{3}^{2} I \text {, } \\
& U_{3}=\alpha_{1}^{3} T_{3} U_{2}+\alpha_{2}^{3} U_{2}+\alpha_{3}^{3} I, \\
& \vdots \\
& U_{N-1}=\alpha_{1}^{N-1} T_{N-1} U_{N-2}+\alpha_{2}^{N-1} U_{N-2}+\alpha_{3}^{N-1} I, \\
& S=U_{N}=\alpha_{1}^{N} T_{N} U_{N-1}+\alpha_{2}^{N} U_{N-1}+\alpha_{3}^{N} I .
\end{aligned}
$$

This mapping is called an S-mapping generated by $T_{1}, \ldots, T_{N}$ and $\alpha_{1}, \alpha_{2}, \ldots, \alpha_{N}$.

The next lemma is very useful for our consideration.

Lemma 2.9 Let $C$ be a nonempty closed convex subset of a real Hilbert space. Let $\left\{T_{i}\right\}_{i=1}^{N}$ be a finite family of nonspreading mappings of $C$ into $C$ with $\bigcap_{i=1}^{N} F\left(T_{i}\right) \neq \emptyset$, and let $\alpha_{j}=$ $\left(\alpha_{1}^{j}, \alpha_{2}^{j}, \alpha_{3}^{j}\right) \in I \times I \times I, j=1,2,3, \ldots, N$, where $I=[0,1], \alpha_{1}^{j}+\alpha_{2}^{j}+\alpha_{3}^{j}=1, \alpha_{1}^{j}, \alpha_{3}^{j} \in(0,1)$ for all $j=1,2, \ldots, N-1$ and $\alpha_{1}^{N} \in(0,1], \alpha_{3}^{N} \in[0,1), \alpha_{2}^{j} \in[0,1)$ for all $j=1,2, \ldots, N$. Let $S$ be the mapping generated by $T_{1}, \ldots, T_{N}$ and $\alpha_{1}, \alpha_{2}, \ldots, \alpha_{N}$. Then $F(S)=\bigcap_{i=1}^{N} F\left(T_{i}\right)$ and $S$ is a quasi-nonexpansive mapping.

Proof It easy to see that $\bigcap_{i=1}^{N} F\left(T_{i}\right) \subseteq F(S)$. Let $x_{0} \in F(S)$ and $x^{*} \in \bigcap_{i=1}^{N} F\left(T_{i}\right)$. Since $\left\{T_{i}\right\}_{i=1}^{N}$ is a finite family of nonspreading mappings of $C$ into itself, for every $y \in C$, we have

$$
\left\|T_{i} y-x^{*}\right\|^{2} \leq \frac{1}{2}\left(\left\|T_{i} y-x^{*}\right\|^{2}+\left\|y-x^{*}\right\|^{2}\right) .
$$

This implies that

$$
\left\|T_{i} y-x^{*}\right\|^{2} \leq\left\|y-x^{*}\right\|^{2}, \quad \forall y \in C \text { and } i=1,2, \ldots, N .
$$


From the definition of $S$ and (2.4),

$$
\begin{aligned}
& \left\|S x_{0}-x^{*}\right\|^{2}=\left\|\alpha_{1}^{N} T_{N} U_{N-1} x_{0}+\alpha_{2}^{N} U_{N-1} x_{0}+\alpha_{3}^{N} x_{0}-x^{*}\right\|^{2} \\
& =\left\|\alpha_{1}^{N}\left(T_{N} U_{N-1} x_{0}-x^{*}\right)+\alpha_{2}^{N}\left(U_{N-1} x_{0}-x^{*}\right)+\alpha_{3}^{N}\left(x_{0}-x^{*}\right)\right\|^{2} \\
& \leq \alpha_{1}^{N}\left\|T_{N} U_{N-1} x_{0}-x^{*}\right\|^{2}+\alpha_{2}^{N}\left\|U_{N-1} x_{0}-x^{*}\right\|^{2}+\alpha_{3}^{N}\left\|x_{0}-x^{*}\right\|^{2} \\
& \leq\left(1-\alpha_{3}^{N}\right)\left\|U_{N-1} x_{0}-x^{*}\right\|^{2}+\alpha_{3}^{N}\left\|x_{0}-x^{*}\right\|^{2} \\
& =\left(1-\alpha_{3}^{N}\right) \| \alpha_{1}^{N-1}\left(T_{N-1} U_{N-2} x_{0}-x^{*}\right)+\alpha_{2}^{N-1}\left(U_{N-2} x_{0}-x^{*}\right) \\
& +\alpha_{3}^{N-1}\left(x_{0}-x^{*}\right)\left\|^{2}+\alpha_{3}^{N}\right\| x_{0}-x^{*} \|^{2} \\
& \leq\left(1-\alpha_{3}^{N}\right)\left(\alpha_{1}^{N-1}\left\|T_{N-1} U_{N-2} x_{0}-x^{*}\right\|^{2}+\alpha_{2}^{N-1}\left\|U_{N-2} x_{0}-x^{*}\right\|^{2}\right. \\
& \left.+\alpha_{3}^{N-1}\left\|x_{0}-x^{*}\right\|^{2}\right)+\alpha_{3}^{N}\left\|x_{0}-x^{*}\right\|^{2} \\
& \leq\left(1-\alpha_{3}^{N}\right)\left(\left(1-\alpha_{3}^{N-1}\right)\left\|U_{N-2} x_{0}-x^{*}\right\|^{2}+\alpha_{3}^{N-1}\left\|x_{0}-x^{*}\right\|^{2}\right) \\
& +\alpha_{3}^{N}\left\|x_{0}-x^{*}\right\|^{2} \\
& =\left(1-\alpha_{3}^{N}\right)\left(1-\alpha_{3}^{N-1}\right)\left\|U_{N-2} x_{0}-x^{*}\right\|^{2}+\alpha_{3}^{N-1}\left(1-\alpha_{3}^{N}\right)\left\|x_{0}-x^{*}\right\|^{2} \\
& +\alpha_{3}^{N}\left\|x_{0}-x^{*}\right\|^{2} \\
& =\prod_{j=N-1}^{N}\left(1-\alpha_{3}^{j}\right)\left\|U_{N-2} x_{0}-x^{*}\right\|^{2}+\left(1-\prod_{j=N-1}^{N}\left(1-\alpha_{3}^{j}\right)\right)\left\|x_{0}-x^{*}\right\|^{2} \\
& \leq \prod_{j=3}^{N}\left(1-\alpha_{3}^{j}\right)\left\|U_{2} x_{0}-x^{*}\right\|^{2}+\left(1-\prod_{j=3}^{N}\left(1-\alpha_{3}^{j}\right)\right)\left\|x_{0}-x^{*}\right\|^{2} \\
& \leq \prod_{j=2}^{N}\left(1-\alpha_{3}^{j}\right)\left\|U_{1} x_{0}-x^{*}\right\|^{2}+\left(1-\prod_{j=2}^{N}\left(1-\alpha_{3}^{j}\right)\right)\left\|x_{0}-x^{*}\right\|^{2} \\
& =\prod_{j=2}^{N}\left(1-\alpha_{3}^{j}\right)\left\|\alpha_{1}^{1}\left(T_{1} x_{0}-x^{*}\right)+\left(1-\alpha_{1}^{1}\right)\left(x_{0}-x^{*}\right)\right\|^{2} \\
& +\left(1-\prod_{j=2}^{N}\left(1-\alpha_{3}^{j}\right)\right)\left\|x_{0}-x^{*}\right\|^{2} \\
& =\prod_{j=2}^{N}\left(1-\alpha_{3}^{j}\right)\left(\alpha_{1}^{1}\left\|T_{1} x_{0}-x^{*}\right\|^{2}+\left(1-\alpha_{1}^{1}\right)\left\|x_{0}-x^{*}\right\|^{2}\right. \\
& \left.-\alpha_{1}^{1}\left(1-\alpha_{1}^{1}\right)\left\|T_{1} x_{0}-x_{0}\right\|\right)+\left(1-\prod_{j=2}^{N}\left(1-\alpha_{3}^{j}\right)\right)\left\|x_{0}-x^{*}\right\|^{2} \\
& \leq \prod_{j=2}^{N}\left(1-\alpha_{3}^{j}\right)\left(\left\|x_{0}-x^{*}\right\|^{2}-\alpha_{1}^{1}\left(1-\alpha_{1}^{1}\right)\left\|T_{1} x_{0}-x_{0}\right\|^{2}\right) \\
& +\left(1-\prod_{j=2}^{N}\left(1-\alpha_{3}^{j}\right)\right)\left\|x_{0}-x^{*}\right\|^{2} .
\end{aligned}
$$


From (2.5), we have

$$
\begin{aligned}
\left\|x_{0}-x^{*}\right\|^{2} \leq & \prod_{j=2}^{N}\left(1-\alpha_{3}^{j}\right)\left(\left\|x_{0}-x^{*}\right\|^{2}-\alpha_{1}^{1}\left(1-\alpha_{1}^{1}\right)\left\|T_{1} x_{0}-x_{0}\right\|^{2}\right) \\
& +\left(1-\prod_{j=2}^{N}\left(1-\alpha_{3}^{j}\right)\right)\left\|x_{0}-x^{*}\right\|^{2},
\end{aligned}
$$

which implies that

$$
\left\|x_{0}-x^{*}\right\|^{2} \leq\left\|x_{0}-x^{*}\right\|^{2}-\alpha_{1}^{1}\left(1-\alpha_{1}^{1}\right)\left\|T_{1} x_{0}-x_{0}\right\|^{2}
$$

Since $\alpha_{1}^{j} \in(0,1)$ for all $j=1,2, \ldots, N-1$ and (2.6), we have $x_{0} \in F\left(T_{1}\right)$. From $x_{0}=T_{1} x_{0}$ and the definition of $S$, we have

$$
U_{1} x_{0}=\alpha_{1}^{1} T_{1} x_{0}+\alpha_{2}^{1} x_{0}+\alpha_{3}^{1} x_{0}=x_{0} .
$$

From (2.5) and $x_{0} \in F\left(U_{1}\right)$, we have

$$
\begin{aligned}
& \left\|x_{0}-x^{*}\right\|^{2} \leq \prod_{j=3}^{N}\left(1-\alpha_{3}^{j}\right)\left\|U_{2} x_{0}-x^{*}\right\|^{2}+\left(1-\prod_{j=3}^{N}\left(1-\alpha_{3}^{j}\right)\right)\left\|x_{0}-x^{*}\right\|^{2} \\
& =\prod_{j=3}^{N}\left(1-\alpha_{3}^{j}\right)\left\|\alpha_{1}^{2} T_{2} U_{1} x_{0}+\alpha_{2}^{2} U_{1} x_{0}+\alpha_{3}^{2} x_{0}-x^{*}\right\|^{2} \\
& +\left(1-\prod_{j=3}^{N}\left(1-\alpha_{3}^{j}\right)\right)\left\|x_{0}-x^{*}\right\|^{2} \\
& =\prod_{j=3}^{N}\left(1-\alpha_{3}^{j}\right)\left\|\alpha_{1}^{2}\left(T_{2} x_{0}-x^{*}\right)+\left(1-\alpha_{1}^{2}\right)\left(x_{0}-x^{*}\right)\right\|^{2} \\
& +\left(1-\prod_{j=3}^{N}\left(1-\alpha_{3}^{j}\right)\right)\left\|x_{0}-x^{*}\right\|^{2} \\
& =\prod_{j=3}^{N}\left(1-\alpha_{3}^{j}\right)\left(\alpha_{1}^{2}\left\|T_{2} x_{0}-x^{*}\right\|^{2}+\left(1-\alpha_{1}^{2}\right)\left\|x_{0}-x^{*}\right\|^{2}\right. \\
& \left.-\alpha_{1}^{2}\left(1-\alpha_{1}^{2}\right)\left\|T_{2} x_{0}-x_{0}\right\|^{2}\right) \\
& +\left(1-\prod_{j=3}^{N}\left(1-\alpha_{3}^{j}\right)\right)\left\|x_{0}-x^{*}\right\|^{2} \\
& \leq \prod_{j=3}^{N}\left(1-\alpha_{3}^{j}\right)\left(\left\|x_{0}-x^{*}\right\|^{2}-\alpha_{1}^{2}\left(1-\alpha_{1}^{2}\right)\left\|T_{2} x_{0}-x_{0}\right\|^{2}\right) \\
& +\left(1-\prod_{j=3}^{N}\left(1-\alpha_{3}^{j}\right)\right)\left\|x_{0}-x^{*}\right\|^{2},
\end{aligned}
$$


which implies that

$$
\left\|x_{0}-x^{*}\right\|^{2} \leq\left\|x_{0}-x^{*}\right\|^{2}-\alpha_{1}^{2}\left(1-\alpha_{1}^{2}\right)\left\|T_{2} x_{0}-x_{0}\right\|^{2}
$$

Since $\alpha_{1}^{j} \in(0,1)$ for all $j=1,2, \ldots, N-1$ and (2.7), we have $x_{0} \in F\left(T_{2}\right)$. From the definition of $S$ and $x_{0}=T_{2} x_{0}$, we have

$$
U_{2} x_{0}=\alpha_{1}^{2} T_{2} U_{1} x_{0}+\alpha_{2}^{2} U_{1} x_{0}+\alpha_{3}^{2} x_{0}=x_{0} .
$$

By continuing in this way, we can show that $x_{0} \in F\left(T_{i}\right)$ and $x_{0} \in F\left(U_{i}\right)$ for all $i=$ $1,2, \ldots, N-1$.

Finally, we shall show that $x_{0} \in F\left(T_{N}\right)$.

Since

$$
\begin{aligned}
0 & =S x_{0}-x_{0}=\alpha_{1}^{N} T_{N} U_{N-1} x_{0}+\alpha_{2}^{N} U_{N-1} x_{0}+\alpha_{3}^{N} x_{0}-x_{0} \\
& =\alpha_{1}^{N}\left(T_{N} x_{0}-x_{0}\right)
\end{aligned}
$$

and $\alpha_{1}^{N} \in(0,1]$, we obtain $T_{N} x_{0}=x_{0}$ so that $x_{0} \in F\left(T_{N}\right)$. Then we have $x_{0} \in \bigcap_{i=1}^{N} F\left(T_{i}\right)$. Hence, $F(S) \subseteq \bigcap_{i=1}^{N} F\left(T_{i}\right)$.

Next, we show that $S$ is a quasi-nonexpansive mapping. Let $x \in C$ and $y \in F(S)$. From (2.5), we can imply that

$$
\begin{aligned}
\|S x-y\|^{2} \leq & \prod_{j=2}^{N}\left(1-\alpha_{3}^{j}\right)\left(\|x-y\|^{2}-\alpha_{1}^{1}\left(1-\alpha_{1}^{1}\right)\left\|T_{1} x-x\right\|\right) \\
& +\left(1-\prod_{j=2}^{N}\left(1-\alpha_{3}^{j}\right)\right)\|x-y\|^{2} \\
\leq & \|x-y\|^{2} .
\end{aligned}
$$

Then we have the $S$-mapping is quasi-nonexpansive.

Example 2.10 Let $T_{1}:[-1,1] \rightarrow[-1,1]$ be a mapping defined by

$$
T_{1} x= \begin{cases}\frac{x+1}{2} & \text { if } x \in(0,1] \\ \frac{-x+1}{2} & \text { if } x \in[-1,0]\end{cases}
$$

for all $x \in[-1,1]$.

Let $T_{2}:[-1,1] \rightarrow[-1,1]$ be a mapping defined by

$$
T_{2} x= \begin{cases}\frac{x+2}{3} & \text { if } x \in(0,1], \\ \frac{-x+2}{3} & \text { if } x \in[-1,0]\end{cases}
$$

for all $x \in[-1,1]$. 
To see that $T_{1}$ is a nonspreading mapping, observe that if $x, y \in(0,1]$, we have $T_{1} x=\frac{x+1}{2}$ and $T_{1} y=\frac{y+1}{2}$. Then we have

$$
\begin{aligned}
\left|T_{1} x-T_{1} y\right|^{2} & =\left|\frac{x+1}{2}-\frac{y+1}{2}\right|^{2} \\
& =\frac{1}{4}|x-y|^{2}
\end{aligned}
$$

and

$$
\begin{aligned}
2\left\langle x-T_{1} x, y-T_{1} y\right\rangle & =2\left\langle x-\left(\frac{x+1}{2}\right), y-\left(\frac{y+1}{2}\right)\right\rangle \\
& =2\left\langle\frac{x-1}{2}, \frac{y-1}{2}\right\rangle \\
& =\frac{1}{2}(x-1)(y-1) \\
& \geq 0 \quad(\text { since } x \leq 1, y \leq 1, \text { then }(x-1)(y-1) \geq 0) .
\end{aligned}
$$

From above, we have

$$
\begin{aligned}
|x-y|^{2}+2\left\langle x-T_{1} x, y-T_{1} y\right\rangle & \geq|x-y|^{2} \\
& \geq \frac{1}{4}|x-y|^{2} \\
& =\left|T_{1} x-T_{1} y\right|^{2} .
\end{aligned}
$$

For every $x, y \in[-1,0]$, we have $T_{1} x=\frac{-x+1}{2}$ and $T_{1} y=\frac{-y+1}{2}$. From the definition of $T_{1}$, we have

$$
\begin{aligned}
\left|T_{1} x-T_{1} y\right|^{2} & =\left|\frac{-x+1}{2}-\left(\frac{-y+1}{2}\right)\right|^{2} \\
& =\left|\frac{y-x}{2}\right|^{2} \\
& =\frac{1}{4}|x-y|^{2}
\end{aligned}
$$

and

$$
\begin{aligned}
2\left\langle x-T_{1} x, y-T_{1} y\right\rangle & =2\left\langle x-\left(\frac{1-x}{2}\right), y-\left(\frac{1-y}{2}\right)\right\rangle \\
& =2\left\langle\frac{3 x-1}{2}, \frac{3 y-1}{2}\right\rangle \\
& =\frac{1}{2}(3 x-1)(3 y-1) \\
& =\frac{1}{2}(3 x(3 y-1)-(3 y-1)) \\
& =\frac{1}{2}(9 x y-3 x-3 y+1) \\
& >0 \quad(\text { since }-1 \leq x, y \leq 0 \text {, then } 9 x y,-3 x,-3 y \geq 0) .
\end{aligned}
$$


From above, we have

$$
\begin{aligned}
|x-y|^{2}+2\left\langle x-T_{1} x, y-T_{1} y\right\rangle & >|x-y|^{2} \\
& \geq \frac{1}{4}|x-y|^{2} \\
& =\left|T_{1} x-T_{1} y\right|^{2} .
\end{aligned}
$$

Finally, for every $x \in(0,1]$ and $y \in[-1,0]$, we have $T_{1} x=\frac{x+1}{2}$ and $T_{1} y=\frac{-y+1}{2}$. From the definition of $T_{1}$, we have

$$
\begin{aligned}
\left|T_{1} x-T_{1} y\right|^{2} & =\left|\frac{x+1}{2}-\frac{-y+1}{2}\right|^{2} \\
& =\frac{1}{4}|x+y|^{2}
\end{aligned}
$$

and

$$
\begin{aligned}
2\left\langle x-T_{1} x, y-T_{1} y\right\rangle & =2\left\langle x-\left(\frac{x+1}{2}\right), y-\left(\frac{-y+1}{2}\right)\right\rangle \\
& =2\left\langle\frac{x-1}{2}, \frac{3 y-1}{2}\right\rangle \\
& =\frac{1}{2}(x-1)(3 y-1) \\
& =\frac{1}{2}(x(3 y-1)-(3 y-1)) \\
& =\frac{1}{2}(3 x y-x-3 y+1) \\
& =\frac{1}{2}(3 y(x-1)+(1-x)) \\
& \geq 0 \quad(\text { since } 0<x \leq 1 \text { and }-1 \leq y \leq 0, \text { then } 3 y(x-1),(1-x) \geq 0) .
\end{aligned}
$$

From above, we have

$$
\begin{aligned}
|x-y|^{2}+2\left\langle x-T_{1} x, y-T_{1} y\right\rangle & \geq|x-y|^{2} \\
& =x^{2}-2 x y+y^{2} \\
& =x^{2}+2 x y+y^{2}-4 x y \\
& \left.\geq x^{2}+2 x y+y^{2} \quad \text { (since }-4 x y \geq 0\right) \\
& =(x+y)^{2} \\
& \geq \frac{1}{4}(x+y)^{2} \\
& =\left|T_{1} x-T_{1} y\right|^{2} .
\end{aligned}
$$

Then for all $x, y \in[-1,1]$, we have

$$
\left|T_{1} x-T_{1} y\right|^{2} \leq|x-y|^{2}+\left\langle x-T_{1} x, y-T_{1} y\right\rangle \text {. }
$$

Hence, we have $T_{1}$ is a nonspreading mapping. 
Next, we show that $T_{2}$ is a nonspreading mapping. Let $x, y \in(0,1]$, then we have $T_{2} x=$ $\frac{x+2}{3}$ and $T_{2} y=\frac{y+2}{3}$. From the definition of $T_{2}$, we have

$$
\begin{aligned}
\left|T_{2} x-T_{2} y\right|^{2} & =\left|\frac{x+2}{3}-\frac{y+2}{3}\right|^{2} \\
& =\frac{1}{9}|x-y|^{2}
\end{aligned}
$$

and

$$
\begin{aligned}
2\left\langle x-T_{2} x, y-T_{2} y\right\rangle & =2\left\langle x-\left(\frac{x+2}{3}\right), y-\left(\frac{y+2}{3}\right)\right\rangle \\
& =2\left\langle\frac{2 x-2}{3}, \frac{2 y-2}{3}\right\rangle \\
& =\frac{8}{9}(x-1)(y-1) \\
& \geq 0 \quad(\text { since } 0<x, y \leq 1 \text {, then }(x-1)(y-1) \geq 0) .
\end{aligned}
$$

From above, we have

$$
\begin{aligned}
|x-y|^{2}+2\left\langle x-T_{2} x, y-T_{2} y\right\rangle & \geq|x-y|^{2} \\
& \geq \frac{1}{9}|x-y|^{2} \\
& =\left|T_{2} x-T_{2} y\right|^{2} .
\end{aligned}
$$

For every $x, y \in[-1,0]$, we have $T_{2} x=\frac{2-x}{3}$ and $T_{2} y=\frac{2-y}{3}$. From the definition of $T_{2}$, we have

$$
\begin{aligned}
\left|T_{2} x-T_{2} y\right|^{2} & =\left|\frac{2-x}{3}-\frac{2-y}{3}\right|^{2} \\
& =\left|\frac{y-x}{3}\right|^{2} \\
& =\frac{1}{9}|x-y|^{2}
\end{aligned}
$$

and

$$
\begin{aligned}
2\left\langle x-T_{2} x, y-T_{2} y\right\rangle & =2\left\langle x-\left(\frac{2-x}{3}\right), y-\left(\frac{2-y}{3}\right)\right\rangle \\
& =2\left\langle\frac{4 x-2}{3}, \frac{4 y-2}{3}\right\rangle \\
& =\frac{8}{9}(2 x-1)(2 y-1) \\
& =\frac{8}{9}(2 x(2 y-1)-(2 y-1)) \\
& =\frac{8}{9}(4 x y-2 x-2 y+1) \\
& >0 \quad(\text { since }-1 \leq x, y \leq 0, \text { then } 4 x y,-2 x,-2 y \geq 0) .
\end{aligned}
$$


From above, we have

$$
\begin{aligned}
|x-y|^{2}+2\left\langle x-T_{2} x, y-T_{2} y\right\rangle & >|x-y|^{2} \\
& \geq \frac{1}{9}|x-y|^{2} \\
& =\left|T_{2} x-T_{2} y\right|^{2} .
\end{aligned}
$$

Finally, for every $x \in(0,1]$ and $y \in[-1,0]$, we have $T_{2} x=\frac{x+2}{3}$ and $T_{2} y=\frac{2-y}{3}$. From the definition of $T_{2}$, we have

$$
\begin{aligned}
\left|T_{2} x-T_{2} y\right|^{2} & =\left|\frac{x+2}{3}-\frac{2-y}{3}\right|^{2} \\
& =\frac{1}{9}|x+y|^{2}
\end{aligned}
$$

and

$$
\begin{aligned}
2\left\langle x-T_{2} x, y-T_{2} y\right\rangle & =2\left\langle x-\left(\frac{x+2}{3}\right), y-\left(\frac{2-y}{3}\right)\right\rangle \\
& =2\left\langle\frac{2 x-2}{3}, \frac{4 y-2}{3}\right\rangle \\
& =\frac{8}{9}(x-1)(2 y-1) \\
& =\frac{8}{9}(x(2 y-1)-(2 y-1)) \\
& =\frac{8}{9}(2 x y-x-2 y+1) \\
& =\frac{8}{9}(2 y(x-1)+(1-x)) \\
& \geq 0 \quad(\text { since } 0<x \leq 1 \text { and }-1 \leq y \leq 0, \text { then } 2 y(x-1),(1-x) \geq 0) .
\end{aligned}
$$

From above, we have

$$
\begin{aligned}
|x-y|^{2}+2\left\langle x-T_{2} x, y-T_{2} y\right\rangle & \geq|x-y|^{2} \\
& =x^{2}-2 x y+y^{2} \\
& =x^{2}+2 x y+y^{2}-4 x y \\
& \geq(x+y)^{2} \quad(\text { since }-4 x y \geq 0) \\
& \geq \frac{1}{9}|x+y|^{2} \\
& =\left|T_{2} x-T_{2} y\right|^{2} .
\end{aligned}
$$

Then for every $x, y \in[-1,1]$, we have

$$
\left|T_{2} x-T_{2} y\right|^{2} \leq|x-y|^{2}+2\left\langle x-T_{2} x, y-T_{2} y\right\rangle .
$$


Hence, we have $T_{2}$ is a nonspreading mapping. Observe that $1 \in F\left(T_{1}\right) \cap F\left(T_{2}\right)$. Let the mapping $S:[-1,1] \rightarrow[-1,1]$ be the $S$-mapping generated by $T_{1}, T_{2}$ and $\alpha_{1}, \alpha_{2}$, where $\alpha_{1}=$ $\left(\frac{1}{6}, \frac{2}{6}, \frac{3}{6}\right)$ and $\left(\frac{4}{15}, \frac{5}{15}, \frac{6}{15}\right)$. From Lemma 2.9 , we have $1 \in F(S)$.

\section{Main result}

Theorem 3.1 Let $C$ be a nonempty closed convex subset of a Hilbert space $H$. For every $i=1,2, \ldots, N$, let $A_{i}: C \rightarrow H$ be an $\alpha_{i}$-inverse strongly monotone mapping, and let $\left\{T_{i}\right\}_{i=1}^{N}$ be a finite family of nonspreading mappings with $\mathfrak{F}=\bigcap_{i=1}^{N} F\left(T_{i}\right) \cap \bigcap_{i=1}^{N} V I\left(C, A_{i}\right) \neq \emptyset$. For every $i=1,2, \ldots, N$, define the mapping $G_{i}: C \rightarrow C$ by $G_{i} x=P_{C}\left(I-\lambda A_{i}\right) x \forall x \in C$ and $\lambda \in$ $[c, d] \subset\left(0,2 \alpha_{i}\right)$. Let $\rho_{j}=\left(\alpha_{1}^{j}, \alpha_{2}^{j}, \alpha_{3}^{j}\right) \in I \times I \times I, j=1,2,3, \ldots, N$, where $I=[0,1], \alpha_{1}^{j}+\alpha_{2}^{j}+$ $\alpha_{3}^{j}=1, \alpha_{1}^{j}, \alpha_{3}^{j} \in(0,1)$ for all $j=1,2, \ldots, N-1$ and $\alpha_{1}^{N} \in(0,1], \alpha_{3}^{N} \in[0,1) \alpha_{2}^{j} \in(0,1)$ for all $j=1,2, \ldots, N$, and let $S$ be the $S$-mapping generated by $T_{1}, T_{2}, \ldots, T_{N}$ and $\rho_{1}, \rho_{2}, \ldots, \rho_{N}$. Let $\left\{x_{n}\right\}$ be a sequence generated by $x_{1} \in C_{1}=C$ and

$$
\left\{\begin{array}{l}
z_{n}=\sum_{i=1}^{N} \delta_{n}^{i} G_{i} x_{n}, \\
y_{n}=\alpha_{n} x_{n}+\beta_{n} S x_{n}+\gamma_{n} z_{n}, \\
C_{n+1}=\left\{z \in C_{n}:\left\|y_{n}-z\right\| \leq\left\|x_{n}-z\right\|\right\}, \\
x_{n+1}=P_{C_{n+1}} x_{1}, \quad \forall n \geq 1,
\end{array}\right.
$$

where $\left\{\alpha_{n}\right\},\left\{\beta_{n}\right\},\left\{\gamma_{n}\right\} \subseteq[0,1], \alpha_{n}+\beta_{n}+\gamma_{n}=1$ and suppose the following conditions hold:

(i) $\lim _{n \rightarrow \infty} \delta_{n}^{i}=\delta^{i} \in(0,1), \quad \forall i=1,2, \ldots, N$ and $\sum_{i=1}^{N} \delta_{n}^{i}=1$,

(ii) $\left\{\alpha_{n}\right\},\left\{\beta_{n}\right\},\left\{\gamma_{n}\right\} \subseteq[a, b] \subset(0,1)$

Then the sequence $\left\{x_{n}\right\}$ converges strongly to $P_{\mathfrak{F}} x_{1}$.

Proof First, we show that $\left(I-\lambda A_{i}\right)$ is a nonexpansive mapping for every $i=1,2, \ldots, N$. Let $x, y \in C$. Since $A$ is an $\alpha_{i}$-inverse strongly monotone and $\lambda<2 \alpha_{i}$, we have

$$
\begin{aligned}
\left\|\left(I-\lambda A_{i}\right) x-\left(I-\lambda A_{i}\right) y\right\|^{2} & =\left\|x-y-\lambda\left(A_{i} x-A_{i} y\right)\right\|^{2} \\
& =\|x-y\|^{2}-2 \lambda\left\langle x-y, A_{i} x-A_{i} y\right\rangle+\lambda^{2}\left\|A_{i} x-A_{i} y\right\|^{2} \\
& \leq\|x-y\|^{2}-2 \alpha_{i} \lambda\left\|A_{i} x-A_{i} y\right\|^{2}+\lambda^{2}\left\|A_{i} x-A_{i} y\right\|^{2} \\
& =\|x-y\|^{2}+\lambda\left(\lambda-2 \alpha_{i}\right)\left\|A_{i} x-A_{i} y\right\|^{2} \\
& \leq\|x-y\|^{2} .
\end{aligned}
$$

Thus $\left(I-\lambda A_{i}\right)$ is a nonexpansive mapping for every $i=1,2, \ldots, N$. Since $P_{C}$ is a nonexpansive mapping, we have $G_{i}$ is a nonexpansive mapping for every $i=1,2, \ldots, N$. From Lemma 2.5, we have

$$
F\left(G_{i}\right)=F\left(P_{C}\left(I-\lambda A_{i}\right)\right)=V I\left(C, A_{i}\right), \quad \forall i=1,2, \ldots, N
$$


From (3.2), $V I\left(C, A_{i}\right)$ is closed and convex. Let $z \in \mathfrak{F}$. From (3.2), we have $z \in F\left(P_{C}\left(I-\lambda A_{i}\right)\right)$ for every $i=1,2, \ldots, N$. By nonexpansiveness of $G_{i}$, we have

$$
\left\|z_{n}-z\right\|=\left\|\sum_{i=1}^{N} \delta_{n}^{i}\left(G_{i} x_{n}-z\right)\right\| \leq \sum_{i=1}^{N} \delta_{n}^{i}\left\|x_{n}-z\right\|=\left\|x_{n}-z\right\| .
$$

Next, we show that $C_{n}$ is closed and convex for every $n \in \mathbb{N}$. It is obvious that $C_{n}$ is closed. In fact, we know that for $z \in C_{n}$,

$$
\left\|y_{n}-z\right\| \leq\left\|x_{n}-z\right\| \quad \text { is equivalent to } \quad\left\|y_{n}-x_{n}\right\|^{2}+2\left\langle y_{n}-x_{n}, x_{n}-z\right\rangle \leq 0 .
$$

So, for every $z_{1}, z_{2} \in C_{n}$ and $t \in(0,1)$, it follows that

$$
\begin{aligned}
& \left\|y_{n}-x_{n}\right\|^{2}+2\left\langle y_{n}-x_{n}, x_{n}-\left(t z_{1}+(1-t) z_{2}\right)\right\rangle \\
& =t\left(2\left\langle y_{n}-x_{n}, x_{n}-z_{1}\right\rangle+\left\|y_{n}-x_{n}\right\|^{2}\right) \\
& \quad+(1-t)\left(2\left\langle y_{n}-x_{n}, x_{n}-z_{2}\right\rangle+\left\|y_{n}-x_{n}\right\|^{2}\right) \\
& \leq 0
\end{aligned}
$$

then, we have $C_{n}$ is convex. Since $V I\left(C, A_{i}\right)$ is closed and convex for every $i=1,2, \ldots, N$, we have $\bigcap_{i=1}^{N} V I\left(C, A_{i}\right)$ is closed and convex. From Lemma 2.4, we have $\bigcap_{i=1}^{N} F\left(T_{i}\right)$ is closed and convex. Hence, we have $\mathfrak{F}$ is closed and convex. This implies that $P_{\mathfrak{F}}$ is well defined. Next, we show that $\mathfrak{F} \subset C_{n}$ for every $n \in \mathbb{N}$. Let $z \in \mathfrak{F}$, then we have

$$
\begin{aligned}
\left\|y_{n}-z\right\| & =\left\|\alpha_{n}\left(x_{n}-z\right)+\beta_{n}\left(S x_{n}-z\right)+\gamma_{n}\left(z_{n}-z\right)\right\| \\
& \leq \alpha_{n}\left\|x_{n}-z\right\|+\beta_{n}\left\|S x_{n}-z\right\|+\gamma_{n}\left\|z_{n}-z\right\| \\
& \leq\left\|x_{n}-z\right\| .
\end{aligned}
$$

It follows that $z \in C_{n}$. Hence, we have $\mathfrak{F} \subset C_{n}$ for every $n \in \mathbb{N}$. This implies that $\left\{x_{n}\right\}$ is well defined. Since $x_{n}=P_{C_{n}} x_{1}$, for every $w \in C_{n}$, we have

$$
\left\|x_{n}-x_{1}\right\| \leq\left\|w-x_{1}\right\|, \quad \forall n \in \mathbb{N} .
$$

In particular, we have

$$
\left\|x_{n}-x_{1}\right\| \leq\left\|P_{\mathfrak{F}} x_{1}-x_{1}\right\|
$$

By (3.4) we have $\left\{x_{n}\right\}$ is bounded, so are $\left\{G_{i} x_{n}\right\},\left\{T_{i} x_{n}\right\}$ for every $i=1,2, \ldots, N,\left\{z_{n}\right\},\left\{y_{n}\right\}$ and $\left\{S x_{n}\right\}$. Since $x_{n+1}=P_{C_{n+1}} x_{1} \in C_{n+1} \subset C_{n}$ and $x_{n}=P_{C_{n}} x_{1}$, we have

$$
\begin{aligned}
0 & \leq\left\langle x_{1}-x_{n}, x_{n}-x_{n+1}\right\rangle \\
& =\left\langle x_{1}-x_{n}, x_{n}-x_{1}+x_{1}-x_{n+1}\right\rangle \\
& \leq-\left\|x_{n}-x_{1}\right\|^{2}+\left\|x_{n}-x_{1}\right\|\left\|x_{1}-x_{n+1}\right\|,
\end{aligned}
$$


which implies that

$$
\left\|x_{n}-x_{1}\right\| \leq\left\|x_{n+1}-x_{1}\right\|
$$

Hence, we have $\lim _{n \rightarrow \infty}\left\|x_{n}-x_{1}\right\|$ exists. Since

$$
\begin{aligned}
\left\|x_{n}-x_{n+1}\right\|^{2} & =\left\|x_{n}-x_{1}+x_{1}-x_{n+1}\right\|^{2} \\
& =\left\|x_{n}-x_{1}\right\|^{2}+2\left\langle x_{n}-x_{1}, x_{1}-x_{n+1}\right\rangle+\left\|x_{1}-x_{n+1}\right\|^{2} \\
& =\left\|x_{n}-x_{1}\right\|^{2}+2\left\langle x_{n}-x_{1}, x_{1}-x_{n}+x_{n}-x_{n+1}\right\rangle+\left\|x_{1}-x_{n+1}\right\|^{2} \\
& =\left\|x_{n}-x_{1}\right\|^{2}-2\left\|x_{n}-x_{1}\right\|^{2}+2\left\langle x_{n}-x_{1}, x_{n}-x_{n+1}\right\rangle+\left\|x_{1}-x_{n+1}\right\|^{2} \\
& \leq\left\|x_{1}-x_{n+1}\right\|^{2}-\left\|x_{n}-x_{1}\right\|^{2},
\end{aligned}
$$

it implies that

$$
\lim _{n \rightarrow \infty}\left\|x_{n}-x_{n+1}\right\|=0 .
$$

Since $x_{n+1}=P_{C_{n+1}} x_{1} \in C_{n+1}$, we have

$$
\left\|y_{n}-x_{n+1}\right\| \leq\left\|x_{n}-x_{n+1}\right\|
$$

By (3.7) we have

$$
\lim _{n \rightarrow \infty}\left\|y_{n}-x_{n+1}\right\|=0 .
$$

Since

$$
\left\|y_{n}-x_{n}\right\| \leq\left\|y_{n}-x_{n+1}\right\|+\left\|x_{n+1}-x_{n}\right\|,
$$

by (3.7) and (3.8), we have

$$
\lim _{n \rightarrow \infty}\left\|y_{n}-x_{n}\right\|=0
$$

Next, we will show that

$$
\lim _{n \rightarrow \infty}\left\|x_{n}-S x_{n}\right\|=0
$$

For every $i=1,2, \ldots, N$, we have

$$
\begin{aligned}
& \left\|P_{C}\left(I-\lambda A_{i}\right) x_{n}-z\right\|^{2} \\
& \quad=\left\|P_{C}\left(I-\lambda A_{i}\right) x_{n}-P_{C}\left(I-\lambda A_{i}\right) z\right\|^{2} \\
& \quad \leq\left\|\left(I-\lambda A_{i}\right) x_{n}-\left(I-\lambda A_{i}\right) z\right\|^{2} \\
& \quad=\left\|x_{n}-z-\lambda\left(A_{i} x_{n}-A_{i} z\right)\right\|^{2} \\
& \quad=\left\|x_{n}-z\right\|^{2}+\lambda^{2}\left\|A_{i} x_{n}-A_{i} z\right\|^{2}-2 \lambda\left\langle x_{n}-z, A_{i} x_{n}-A_{i} z\right\rangle
\end{aligned}
$$




$$
\begin{aligned}
& \leq\left\|x_{n}-z\right\|^{2}+\lambda^{2}\left\|A_{i} x_{n}-A_{i} z\right\|^{2}-2 \lambda \alpha_{i}\left\|A_{i} x_{n}-A_{i} z\right\|^{2} \\
& =\left\|x_{n}-z\right\|^{2}-\lambda\left(2 \alpha_{i}-\lambda\right)\left\|A_{i} x_{n}-A_{i} z\right\|^{2} .
\end{aligned}
$$

From the definition of $y_{n}$ and (3.11), we have

$$
\begin{aligned}
\left\|y_{n}-z\right\|^{2} \leq & \alpha_{n}\left\|x_{n}-z\right\|^{2}+\beta_{n}\left\|S x_{n}-z\right\|^{2}+\gamma_{n}\left\|z_{n}-z\right\|^{2} \\
\leq & \alpha_{n}\left\|x_{n}-z\right\|^{2}+\beta_{n}\left\|S x_{n}-z\right\|^{2}+\gamma_{n} \sum_{i=1}^{N} \delta_{n}^{i}\left\|P_{C}\left(I-\lambda A_{i}\right) x_{n}-z\right\|^{2} \\
\leq & \alpha_{n}\left\|x_{n}-z\right\|^{2}+\beta_{n}\left\|S x_{n}-z\right\|^{2} \\
& +\gamma_{n} \sum_{i=1}^{N} \delta_{n}^{i}\left(\left\|x_{n}-z\right\|^{2}-\lambda\left(2 \alpha_{i}-\lambda\right)\left\|A_{i} x_{n}-A_{i} z\right\|^{2}\right) \\
= & \alpha_{n}\left\|x_{n}-z\right\|^{2}+\beta_{n}\left\|S x_{n}-z\right\|^{2}+\gamma_{n}\left\|x_{n}-z\right\|^{2} \\
& -\gamma_{n} \sum_{i=1}^{N} \delta_{n}^{i} \lambda\left(2 \alpha_{i}-\lambda\right)\left\|A_{i} x_{n}-A_{i} z\right\|^{2} \\
\leq & \left\|x_{n}-z\right\|^{2}-\gamma_{n} \sum_{i=1}^{N} \delta_{n}^{i} \lambda\left(2 \alpha_{i}-\lambda\right)\left\|A_{i} x_{n}-A_{i} z\right\|^{2} .
\end{aligned}
$$

It follows that

$$
\begin{aligned}
\gamma_{n} \sum_{i=1}^{N} \delta_{n}^{i} \lambda\left(2 \alpha_{i}-\lambda\right)\left\|A_{i} x_{n}-A_{i} z\right\|^{2} & \leq\left\|x_{n}-z\right\|^{2}-\left\|y_{n}-z\right\|^{2} \\
& \leq\left(\left\|x_{n}-z\right\|+\left\|y_{n}-z\right\|\right)\left\|y_{n}-x_{n}\right\| .
\end{aligned}
$$

From conditions (i), (ii) and (3.9), it implies that

$$
\lim _{n \rightarrow \infty}\left\|A_{i} x_{n}-A_{i} z\right\|=0, \quad \forall i=1,2, \ldots, N
$$

Since

$$
\begin{aligned}
\left\|P_{C}\left(I-\lambda A_{i}\right) x_{n}-z\right\|^{2} \leq & \left\langle\left(I-\lambda A_{i}\right) x_{n}-\left(I-\lambda A_{i}\right) z, P_{C}\left(I-\lambda A_{i}\right) x_{n}-z\right\rangle \\
= & \frac{1}{2}\left(\left\|\left(I-\lambda A_{i}\right) x_{n}-\left(I-\lambda A_{i}\right) z\right\|^{2}+\left\|P_{C}\left(I-\lambda A_{i}\right) x_{n}-z\right\|^{2}\right. \\
& \left.-\left\|\left(I-\lambda A_{i}\right) x_{n}-\left(I-\lambda A_{i}\right) z-P_{C}\left(I-\lambda A_{i}\right) x_{n}+z\right\|^{2}\right) \\
\leq & \frac{1}{2}\left(\left\|x_{n}-z\right\|^{2}+\left\|P_{C}\left(I-\lambda A_{i}\right) x_{n}-z\right\|^{2}\right. \\
& \left.-\left\|x_{n}-P_{C}\left(I-\lambda A_{i}\right) x_{n}-\lambda\left(A_{i} x_{n}-A_{i} z\right)\right\|^{2}\right) \\
= & \frac{1}{2}\left(\left\|x_{n}-z\right\|^{2}+\left\|P_{C}\left(I-\lambda A_{i}\right) x_{n}-z\right\|^{2}\right. \\
& -\left\|x_{n}-P_{C}\left(I-\lambda A_{i}\right) x_{n}\right\|^{2}-\left\|\lambda\left(A_{i} x_{n}-A_{i} z\right)\right\|^{2} \\
& \left.+2 \lambda\left(x_{n}-P_{C}\left(I-\lambda A_{i}\right) x_{n}, A_{i} x_{n}-A_{i} z\right)\right),
\end{aligned}
$$


it implies that

$$
\begin{aligned}
\left\|P_{C}\left(I-\lambda A_{i}\right) x_{n}-z\right\|^{2} \leq & \left\|x_{n}-z\right\|^{2}-\left\|x_{n}-P_{C}\left(I-\lambda A_{i}\right) x_{n}\right\|^{2} \\
& +2 \lambda\left\|x_{n}-P_{C}\left(I-\lambda A_{i}\right) x_{n}\right\|\left\|A_{i} x_{n}-A_{i} z\right\| .
\end{aligned}
$$

From the definition of $y_{n}$ and (3.13), we have

$$
\begin{aligned}
\left\|y_{n}-z\right\|^{2} \leq & \alpha_{n}\left\|x_{n}-z\right\|^{2}+\beta_{n}\left\|S x_{n}-z\right\|^{2}+\gamma_{n}\left\|z_{n}-z\right\|^{2} \\
\leq & \left(1-\gamma_{n}\right)\left\|x_{n}-z\right\|^{2}+\gamma_{n} \sum_{i=1}^{N} \delta_{n}^{i}\left\|P_{C}\left(I-\lambda A_{i}\right) x_{n}-z\right\|^{2} \\
\leq & \left(1-\gamma_{n}\right)\left\|x_{n}-z\right\|^{2}+\gamma_{n} \sum_{i=1}^{N} \delta_{n}^{i}\left(\left\|x_{n}-z\right\|^{2}-\left\|x_{n}-P_{C}\left(I-\lambda A_{i}\right) x_{n}\right\|^{2}\right. \\
& \left.+2 \lambda\left\|x_{n}-P_{C}\left(I-\lambda A_{i}\right) x_{n}\right\|\left\|A_{i} x_{n}-A_{i} z\right\|\right) \\
= & \left\|x_{n}-z\right\|^{2}-\gamma_{n} \sum_{i=1}^{N} \delta_{n}^{i}\left\|x_{n}-P_{C}\left(I-\lambda A_{i}\right) x_{n}\right\|^{2} \\
& +2 \gamma_{n} \sum_{i=1}^{N} \delta_{n}^{i} \lambda\left\|x_{n}-P_{C}\left(I-\lambda A_{i}\right) x_{n}\right\|\left\|A_{i} x_{n}-A_{i} z\right\|,
\end{aligned}
$$

which implies that

$$
\begin{aligned}
\gamma_{n} \sum_{i=1}^{N} \delta_{n}^{i}\left\|x_{n}-P_{C}\left(I-\lambda A_{i}\right) x_{n}\right\|^{2} \leq & \left\|x_{n}-z\right\|^{2}-\left\|y_{n}-z\right\|^{2} \\
& +2 \gamma_{n} \sum_{i=1}^{N} \delta_{n}^{i} \lambda\left\|x_{n}-P_{C}\left(I-\lambda A_{i}\right) x_{n}\right\|\left\|A_{i} x_{n}-A_{i} z\right\| \\
\leq & \left(\left\|x_{n}-z\right\|+\left\|y_{n}-z\right\|\right)\left\|y_{n}-x_{n}\right\| \\
& +2 \gamma_{n} \sum_{i=1}^{N} \delta_{n}^{i} \lambda\left\|x_{n}-P_{C}\left(I-\lambda A_{i}\right) x_{n}\right\|\left\|A_{i} x_{n}-A_{i} z\right\| .
\end{aligned}
$$

From conditions (i), (ii), (3.9) and (3.12), we have

$$
\lim _{n \rightarrow \infty}\left\|P_{C}\left(I-\lambda A_{i}\right) x_{n}-x_{n}\right\|=0, \quad \forall i=1,2, \ldots, N
$$

Since

$$
\left\|z_{n}-x_{n}\right\| \leq \sum_{i=1}^{N} \delta_{n}^{i}\left\|P_{C}\left(I-\lambda A_{i}\right) x_{n}-x_{n}\right\|,
$$

from (3.14), we have

$$
\lim _{n \rightarrow \infty}\left\|z_{n}-x_{n}\right\|=0 .
$$


Since

$$
y_{n}-x_{n}=\beta_{n}\left(S x_{n}-x_{n}\right)+\gamma_{n}\left(z_{n}-x_{n}\right)
$$

from (3.9) and (3.15), we have

$$
\lim _{n \rightarrow \infty}\left\|S x_{n}-x_{n}\right\|=0
$$

Next, we will show that

$$
\lim _{n \rightarrow \infty}\left\|T_{i} U_{i-1} x_{n}-U_{i-1} x_{n}\right\|=0, \quad \forall i=1,2, \ldots, N
$$

From the definition of $y_{n}$, we have

$$
\begin{aligned}
& \left\|y_{n}-z\right\|^{2} \leq \alpha_{n}\left\|x_{n}-z\right\|^{2}+\beta_{n}\left\|S x_{n}-z\right\|^{2}+\gamma_{n}\left\|z_{n}-z\right\|^{2} \\
& \leq\left(1-\beta_{n}\right)\left\|x_{n}-z\right\|^{2}+\beta_{n} \| \alpha_{1}^{N}\left(T_{N} U_{N-1} x_{n}-z\right) \\
& +\alpha_{2}^{N}\left(U_{N-1} x_{n}-z\right)+\alpha_{3}^{N}\left(x_{n}-z\right) \| \\
& \leq\left(1-\beta_{n}\right)\left\|x_{n}-z\right\|^{2}+\beta_{n}\left(\alpha_{1}^{N}\left\|T_{N} U_{N-1} x_{n}-z\right\|^{2}+\alpha_{2}^{N}\left\|U_{N-1} x_{n}-z\right\|^{2}\right. \\
& \left.+\alpha_{3}^{N}\left\|x_{n}-z\right\|^{2}-\alpha_{1}^{N} \alpha_{2}^{N}\left\|T_{N} U_{N-1} x_{n}-U_{N-1} x_{n}\right\|^{2}\right) \\
& \leq\left(1-\beta_{n}\right)\left\|x_{n}-z\right\|^{2}+\beta_{n}\left(\left(1-\alpha_{3}^{N}\right)\left\|U_{N-1} x_{n}-z\right\|^{2}\right. \\
& \left.+\alpha_{3}^{N}\left\|x_{n}-z\right\|^{2}-\alpha_{1}^{N} \alpha_{2}^{N}\left\|T_{N} U_{N-1} x_{n}-U_{N-1} x_{n}\right\|^{2}\right) \\
& =\left(1-\beta_{n}\right)\left\|x_{n}-z\right\|^{2}+\beta_{n}\left(\left(1-\alpha_{3}^{N}\right) \| \alpha_{1}^{N-1}\left(T_{N-1} U_{N-2} x_{n}-z\right)\right. \\
& +\alpha_{2}^{N-1}\left(U_{N-2} x_{n}-z\right)+\alpha_{3}^{N-1}\left(x_{n}-z\right) \|^{2} \\
& \left.+\alpha_{3}^{N}\left\|x_{n}-z\right\|^{2}-\alpha_{1}^{N} \alpha_{2}^{N}\left\|T_{N} U_{N-1} x_{n}-U_{N-1} x_{n}\right\|^{2}\right) \\
& \leq\left(1-\beta_{n}\right)\left\|x_{n}-z\right\|^{2}+\beta_{n}\left(( 1 - \alpha _ { 3 } ^ { N } ) \left(\alpha_{1}^{N-1}\left\|T_{N-1} U_{N-2} x_{n}-z\right\|^{2}\right.\right. \\
& +\alpha_{2}^{N-1}\left\|U_{N-2} x_{n}-z\right\|^{2}+\alpha_{3}^{N-1}\left\|x_{n}-z\right\|^{2} \\
& \left.-\alpha_{1}^{N-1} \alpha_{2}^{N-1}\left\|T_{N-1} U_{N-2} x_{n}-U_{N-2} x_{n}\right\|^{2}\right) \\
& \left.+\alpha_{3}^{N}\left\|x_{n}-z\right\|^{2}-\alpha_{1}^{N} \alpha_{2}^{N}\left\|T_{N} U_{N-1} x_{n}-U_{N-1} x_{n}\right\|^{2}\right) \\
& \leq\left(1-\beta_{n}\right)\left\|x_{n}-z\right\|^{2}+\beta_{n}\left(( 1 - \alpha _ { 3 } ^ { N } ) \left(\left(1-\alpha_{3}^{N-1}\right)\left\|U_{N-2} x_{n}-z\right\|^{2}\right.\right. \\
& \left.+\alpha_{3}^{N-1}\left\|x_{n}-z\right\|^{2}-\alpha_{1}^{N-1} \alpha_{2}^{N-1}\left\|T_{N-1} U_{N-2} x_{n}-U_{N-2} x_{n}\right\|^{2}\right) \\
& \left.+\alpha_{3}^{N}\left\|x_{n}-z\right\|^{2}-\alpha_{1}^{N} \alpha_{2}^{N}\left\|T_{N} U_{N-1} x_{n}-U_{N-1} x_{n}\right\|^{2}\right) \\
& =\left(1-\beta_{n}\right)\left\|x_{n}-z\right\|^{2}+\beta_{n}\left(\left(1-\alpha_{3}^{N}\right)\left(1-\alpha_{3}^{N-1}\right)\left\|U_{N-2} x_{n}-z\right\|^{2}\right. \\
& +\left(1-\alpha_{3}^{N}\right) \alpha_{3}^{N-1}\left\|x_{n}-z\right\|^{2}-\alpha_{1}^{N-1} \alpha_{2}^{N-1}\left(1-\alpha_{3}^{N}\right)\left\|T_{N-1} U_{N-2} x_{n}-U_{N-2} x_{n}\right\|^{2} \\
& \left.+\alpha_{3}^{N}\left\|x_{n}-z\right\|^{2}-\alpha_{1}^{N} \alpha_{2}^{N}\left\|T_{N} U_{N-1} x_{n}-U_{N-1} x_{n}\right\|^{2}\right) \\
& =\left(1-\beta_{n}\right)\left\|x_{n}-z\right\|^{2}+\beta_{n}\left(\prod_{j=N-1}^{N}\left(1-\alpha_{3}^{j}\right)\left\|U_{N-2} x_{n}-z\right\|^{2}\right. \\
& +\left(1-\prod_{j=N-1}^{N}\left(1-\alpha_{3}^{j}\right)\right)\left\|x_{n}-z\right\|^{2}
\end{aligned}
$$




$$
\begin{aligned}
& -\alpha_{1}^{N-1} \alpha_{2}^{N-1}\left(1-\alpha_{3}^{N}\right)\left\|T_{N-1} U_{N-2} x_{n}-U_{N-2} x_{n}\right\|^{2} \\
& \left.-\alpha_{1}^{N} \alpha_{2}^{N}\left\|T_{N} U_{N-1} x_{n}-U_{N-1} x_{n}\right\|^{2}\right) \\
& =\left(1-\beta_{n}\right)\left\|x_{n}-z\right\|^{2}+\beta_{n}\left(\prod_{j=N-1}^{N}\left(1-\alpha_{3}^{j}\right) \| \alpha_{1}^{N-2}\left(T_{N-2} U_{N-3} x_{n}-z\right)\right. \\
& +\alpha_{2}^{N-2}\left(U_{N-3} x_{n}-z\right)+\alpha_{3}^{N-2}\left(x_{n}-z\right) \|^{2} \\
& +\left(1-\prod_{j=N-1}^{N}\left(1-\alpha_{3}^{j}\right)\right)\left\|x_{n}-z\right\|^{2} \\
& -\alpha_{1}^{N-1} \alpha_{2}^{N-1}\left(1-\alpha_{3}^{N}\right)\left\|T_{N-1} U_{N-2} x_{n}-U_{N-2} x_{n}\right\|^{2} \\
& \left.-\alpha_{1}^{N} \alpha_{2}^{N}\left\|T_{N} U_{N-1} x_{n}-U_{N-1} x_{n}\right\|^{2}\right) \\
& \leq\left(1-\beta_{n}\right)\left\|x_{n}-z\right\|^{2}+\beta_{n}\left(\prod _ { j = N - 1 } ^ { N } ( 1 - \alpha _ { 3 } ^ { j } ) \left(\alpha_{1}^{N-2}\left\|T_{N-2} U_{N-3} x_{n}-z\right\|^{2}\right.\right. \\
& +\alpha_{2}^{N-2}\left\|U_{N-3} x_{n}-z\right\|^{2}+\alpha_{3}^{N-2}\left\|x_{n}-z\right\|^{2} \\
& \left.-\alpha_{1}^{N-2} \alpha_{2}^{N-2}\left\|T_{N-2} U_{N-3} x_{n}-U_{N-3} x_{n}\right\|^{2}\right) \\
& +\left(1-\prod_{j=N-1}^{N}\left(1-\alpha_{3}^{j}\right)\right)\left\|x_{n}-z\right\|^{2} \\
& -\alpha_{1}^{N-1} \alpha_{2}^{N-1}\left(1-\alpha_{3}^{N}\right)\left\|T_{N-1} U_{N-2} x_{n}-U_{N-2} x_{n}\right\|^{2} \\
& \left.-\alpha_{1}^{N} \alpha_{2}^{N}\left\|T_{N} U_{N-1} x_{n}-U_{N-1} x_{n}\right\|^{2}\right) \\
& \leq\left(1-\beta_{n}\right)\left\|x_{n}-z\right\|^{2}+\beta_{n}\left(\prod _ { j = N - 1 } ^ { N } ( 1 - \alpha _ { 3 } ^ { j } ) \left(\left(1-\alpha_{3}^{N-2}\right)\left\|U_{N-3} x_{n}-z\right\|^{2}\right.\right. \\
& \left.+\alpha_{3}^{N-2}\left\|x_{n}-z\right\|^{2}-\alpha_{1}^{N-2} \alpha_{2}^{N-2}\left\|T_{N-2} U_{N-3} x_{n}-U_{N-3} x_{n}\right\|^{2}\right) \\
& +\left(1-\prod_{j=N-1}^{N}\left(1-\alpha_{3}^{j}\right)\right)\left\|x_{n}-z\right\|^{2} \\
& -\alpha_{1}^{N-1} \alpha_{2}^{N-1}\left(1-\alpha_{3}^{N}\right)\left\|T_{N-1} U_{N-2} x_{n}-U_{N-2} x_{n}\right\|^{2} \\
& \left.-\alpha_{1}^{N} \alpha_{2}^{N}\left\|T_{N} U_{N-1} x_{n}-U_{N-1} x_{n}\right\|^{2}\right) \\
& =\left(1-\beta_{n}\right)\left\|x_{n}-z\right\|^{2}+\beta_{n}\left(\prod_{j=N-2}^{N}\left(1-\alpha_{3}^{j}\right)\left\|U_{N-3} x_{n}-z\right\|^{2}\right. \\
& +\left(1-\prod_{j=N-2}^{N}\left(1-\alpha_{3}^{j}\right)\right)\left\|x_{n}-z\right\|^{2} \\
& -\alpha_{1}^{N-2} \alpha_{2}^{N-2} \prod_{j=N-1}^{N}\left(1-\alpha_{3}^{j}\right)\left\|T_{N-2} U_{N-3} x_{n}-U_{N-3} x_{n}\right\|^{2}
\end{aligned}
$$




$$
\begin{aligned}
& -\alpha_{1}^{N-1} \alpha_{2}^{N-1}\left(1-\alpha_{3}^{N}\right)\left\|T_{N-1} U_{N-2} x_{n}-U_{N-2} x_{n}\right\|^{2} \\
& \left.-\alpha_{1}^{N} \alpha_{2}^{N}\left\|T_{N} U_{N-1} x_{n}-U_{N-1} x_{n}\right\|^{2}\right) \\
& \leq \\
& \leq\left(1-\beta_{n}\right)\left\|x_{n}-z\right\|^{2}+\beta_{n}\left(\prod_{j=1}^{N}\left(1-\alpha_{3}^{j}\right)\left\|U_{0} x_{n}-z\right\|^{2}\right. \\
& +\left(1-\prod_{j=1}^{N}\left(1-\alpha_{3}^{j}\right)\right)\left\|x_{n}-z\right\|^{2} \\
& -\alpha_{1}^{1} \alpha_{2}^{1} \prod_{j=2}^{N}\left(1-\alpha_{3}^{j}\right)\left\|T_{1} U_{0} x_{n}-U_{0} x_{n}\right\|^{2} \\
& \vdots \\
& -\alpha_{1}^{N-2} \alpha_{2}^{N-2} \prod_{j=N-1}^{N}\left(1-\alpha_{3}^{j}\right)\left\|T_{N-2} U_{N-3} x_{n}-U_{N-3} x_{n}\right\|^{2} \\
& -\alpha_{1}^{N-1} \alpha_{2}^{N-1}\left(1-\alpha_{3}^{N}\right)\left\|T_{N-1} U_{N-2} x_{n}-U_{N-2} x_{n}\right\|^{2} \\
& \left.-\alpha_{1}^{N} \alpha_{2}^{N}\left\|T_{N} U_{N-1} x_{n}-U_{N-1} x_{n}\right\|^{2}\right) \\
& =\left\|x_{n}-z\right\|^{2} \\
& -\beta_{n} \alpha_{1}^{1} \alpha_{2}^{1} \prod_{j=2}^{N}\left(1-\alpha_{3}^{j}\right)\left\|T_{1} x_{n}-x_{n}\right\|^{2} \\
& \vdots \\
& -\beta_{n} \alpha_{1}^{N-2} \alpha_{2}^{N-2} \prod_{j=N-1}^{N}\left(1-\alpha_{3}^{j}\right)\left\|T_{N-2} U_{N-3} x_{n}-U_{N-3} x_{n}\right\|^{2} \\
& -\beta_{n} \alpha_{1}^{N-1} \alpha_{2}^{N-1}\left(1-\alpha_{3}^{N}\right)\left\|T_{N-1} U_{N-2} x_{n}-U_{N-2} x_{n}\right\|^{2} \\
& -\beta_{n} \alpha_{1}^{N} \alpha_{2}^{N}\left\|T_{N} U_{N-1} x_{n}-U_{N-1} x_{n}\right\|^{2} \text {. }
\end{aligned}
$$

From (3.17) and condition (ii), we have

$$
\begin{aligned}
\beta_{n} \alpha_{1}^{1} \alpha_{2}^{1} \prod_{j=2}^{N}\left(1-\alpha_{3}^{j}\right)\left\|T_{1} x_{n}-x_{n}\right\|^{2} & \leq\left\|x_{n}-z\right\|^{2}-\left\|y_{n}-z\right\|^{2} \\
& \leq\left(\left\|x_{n}-z\right\|+\left\|y_{n}-z\right\|\right)\left\|y_{n}-x_{n}\right\| .
\end{aligned}
$$

Form (3.9), we have

$$
\lim _{n \rightarrow \infty}\left\|T_{1} x_{n}-x_{n}\right\|=0
$$


By using the same method as (3.18), we can conclude that

$$
\lim _{n \rightarrow \infty}\left\|T_{i} U_{i-1} x_{n}-U_{i-1} x_{n}\right\|=0, \quad \forall i=1,2, \ldots, N
$$

Let $\omega\left(x_{n}\right)$ be the set of all weakly $\omega$-limit of $\left\{x_{n}\right\}$. We shall show that $\omega\left(x_{n}\right) \subset \mathfrak{F}$. Since $\left\{x_{n}\right\}$ is bounded, then $\omega\left(x_{n}\right) \neq \emptyset$. Let $q \in \omega\left(x_{n}\right)$, there exists a subsequence $\left\{x_{n_{i}}\right\}$ of $\left\{x_{n}\right\}$ which converges weakly to $q$.

Put $Q: C \rightarrow C$ defined by

$$
Q x=\sum_{i=1}^{N} \delta^{i} G_{i} x, \quad \forall x \in C .
$$

Since $G_{i}=P_{C}\left(I-\lambda A_{i}\right)$ is a nonexpansive mapping, for every $i=1,2, \ldots, N$, from Lemma 2.6 and 2.5 , we have

$$
F(Q)=\bigcap_{i=1}^{N} F\left(G_{i}\right)=\bigcap_{i=1}^{N} V I\left(C, A_{i}\right) .
$$

Since

$$
\begin{aligned}
\left\|x_{n}-Q x_{n}\right\| & \leq\left\|x_{n}-z_{n}\right\|+\left\|z_{n}-Q x_{n}\right\| \\
& =\left\|x_{n}-z_{n}\right\|+\left\|\sum_{i=1}^{N} \delta_{n}^{i} G_{i} x_{n}-\sum_{i=1}^{N} \delta^{i} G_{i} x_{n}\right\| \\
& =\left\|x_{n}-z_{n}\right\|+\left\|\sum_{i=1}^{N}\left(\delta_{n}^{i}-\delta^{i}\right) G_{i} x_{n}\right\| \\
& \leq\left\|x_{n}-z_{n}\right\|+\sum_{i=1}^{N}\left|\delta_{n}^{i}-\delta^{i}\right|\left\|G_{i} x_{n}\right\|,
\end{aligned}
$$

from the condition (i) and (3.15), we have

$$
\lim _{n \rightarrow \infty}\left\|x_{n}-Q x_{n}\right\|=0
$$

From (3.21), we have

$$
\lim _{i \rightarrow \infty}\left\|x_{n_{i}}-Q x_{n_{i}}\right\|=0
$$

From (3.19), it is easy to see that $Q$ is a nonexpansive mapping. By Lemma 2.7 and $x_{n_{i}} \rightarrow q$ as $i \rightarrow \infty$, we have $q \in F(Q)=\bigcap_{i=1}^{N} F\left(G_{i}\right)$ From (3.2), we have

$$
q \in \bigcap_{i=1}^{N} V I\left(C, A_{i}\right)
$$

Next, we will show that $q \in F(S)$. Assume that $q \neq S q$. From the Opial property, (3.10) and 
(3.16), we have

$$
\begin{aligned}
& \liminf _{i \rightarrow \infty}\left\|x_{n_{i}}-q\right\|^{2}<\liminf _{i \rightarrow \infty}\left\|x_{n_{i}}-S q\right\|^{2} \\
& =\liminf _{i \rightarrow \infty}\left\|x_{n_{i}}-S x_{n_{i}}+\left(S x_{n_{i}}-S q\right)\right\|^{2} \\
& =\liminf _{i \rightarrow \infty}\left(\left\|x_{n_{i}}-S x_{n_{i}}\right\|^{2}+\left\|S x_{n_{i}}-S q\right\|^{2}+2\left\langle x_{n_{i}}-S x_{n_{i}}, S x_{n_{i}}-S q\right\rangle\right) \\
& =\liminf _{i \rightarrow \infty}\left\|S x_{n_{i}}-S q\right\|^{2} \\
& =\liminf _{i \rightarrow \infty} \| \alpha_{1}^{N} T_{N} U_{N-1} x_{n_{i}}+\alpha_{2}^{N} U_{N-1} x_{n_{i}}+\alpha_{3}^{N} x_{n_{i}} \\
& -\alpha_{1}^{N} T_{N} U_{N-1} q-\alpha_{2}^{N} U_{N-1} q-\alpha_{3}^{N} q \|^{2} \\
& =\liminf _{i \rightarrow \infty} \| \alpha_{1}^{N}\left(T_{N} U_{N-1} x_{n_{i}}-T_{N} U_{N-1} q\right) \\
& +\alpha_{2}^{N}\left(U_{N-1} x_{n_{i}}-U_{N-1} q\right)+\alpha_{3}^{N}\left(x_{n_{i}}-q\right) \|^{2} \\
& \leq \liminf _{i \rightarrow \infty}\left(\alpha_{1}^{N}\left\|T_{N} U_{N-1} x_{n_{i}}-T_{N} U_{N-1} q\right\|^{2}\right. \\
& \left.+\alpha_{2}^{N}\left\|U_{N-1} x_{n_{i}}-U_{N-1} q\right\|^{2}+\alpha_{3}^{N}\left\|x_{n_{i}}-q\right\|^{2}\right) \\
& \leq \liminf _{i \rightarrow \infty}\left(\alpha _ { 1 } ^ { N } \left(\left\|U_{N-1} x_{n_{i}}-U_{N-1} q\right\|^{2}\right.\right. \\
& \left.+2\left\langle U_{N-1} x_{n_{i}}-T_{N} U_{N-1} x_{n_{i}}, U_{N-1} q-T_{N} U_{N-1} q\right\rangle\right) \\
& \left.+\alpha_{2}^{N}\left\|U_{N-1} x_{n_{i}}-U_{N-1} q\right\|^{2}+\alpha_{3}^{N}\left\|x_{n_{i}}-q\right\|^{2}\right) \\
& =\liminf _{i \rightarrow \infty}\left(\left(1-\alpha_{3}^{N}\right)\left\|U_{N-1} x_{n_{i}}-U_{N-1} q\right\|^{2}+\alpha_{3}^{N}\left\|x_{n_{i}}-q\right\|^{2}\right) \\
& =\liminf _{i \rightarrow \infty}\left(\left(1-\alpha_{3}^{N}\right) \| \alpha_{1}^{N-1}\left(T_{N-1} U_{N-2} x_{n_{i}}-T_{N-1} U_{N-2} q\right)\right. \\
& \left.+\alpha_{2}^{N-1}\left(U_{N-2} x_{n_{i}}-U_{N-2} q\right)+\alpha_{3}^{N-1}\left(x_{n_{i}}-q\right)\left\|^{2}+\alpha_{3}^{N}\right\| x_{n_{i}}-q \|^{2}\right) \\
& \leq \liminf _{i \rightarrow \infty}\left(( 1 - \alpha _ { 3 } ^ { N } ) \left(\alpha_{1}^{N-1}\left\|T_{N-1} U_{N-2} x_{n_{i}}-T_{N-1} U_{N-2} q\right\|^{2}\right.\right. \\
& \left.\left.+\alpha_{2}^{N-1}\left\|U_{N-2} x_{n_{i}}-U_{N-2} q\right\|^{2}+\alpha_{3}^{N-1}\left\|x_{n_{i}}-q\right\|^{2}\right)+\alpha_{3}^{N}\left\|x_{n_{i}}-q\right\|^{2}\right) \\
& \leq \liminf _{i \rightarrow \infty}\left(( 1 - \alpha _ { 3 } ^ { N } ) \left(\alpha _ { 1 } ^ { N - 1 } \left(\left\|U_{N-2} x_{n_{i}}-U_{N-2} q\right\|^{2}\right.\right.\right. \\
& \left.+2\left\langle U_{N-2} x_{n_{i}}-T_{N-1} U_{N-2} x_{n_{i}}, U_{N-2} q-T_{N-1} U_{N-2} q\right\rangle\right) \\
& \left.\left.+\alpha_{2}^{N-1}\left\|U_{N-2} x_{n_{i}}-U_{N-2} q\right\|^{2}+\alpha_{3}^{N-1}\left\|x_{n_{i}}-q\right\|^{2}\right)+\alpha_{3}^{N}\left\|x_{n_{i}}-q\right\|^{2}\right) \\
& =\liminf _{i \rightarrow \infty}\left(( 1 - \alpha _ { 3 } ^ { N } ) \left(\left(1-\alpha_{3}^{N-1}\right)\left\|U_{N-2} x_{n_{i}}-U_{N-2} q\right\|^{2}\right.\right. \\
& \left.\left.+\alpha_{3}^{N-1}\left\|x_{n_{i}}-q\right\|^{2}\right)+\alpha_{3}^{N}\left\|x_{n_{i}}-q\right\|^{2}\right) \\
& =\liminf _{i \rightarrow \infty}\left(\prod_{j=N-1}^{N}\left(1-\alpha_{3}^{j}\right)\left\|U_{N-2} x_{n_{i}}-U_{N-2} q\right\|^{2}\right. \\
& \left.+\left(1-\prod_{j=N-1}^{N}\left(1-\alpha_{3}^{j}\right)\right)\left\|x_{n_{i}}-q\right\|^{2}\right) \\
& \leq \\
& \vdots
\end{aligned}
$$




$$
\begin{aligned}
& \leq \liminf _{i \rightarrow \infty}\left(\prod_{j=1}^{N}\left(1-\alpha_{3}^{j}\right)\left\|U_{0} x_{n_{i}}-U_{0} q\right\|^{2}\right. \\
& \left.+\left(1-\prod_{j=1}^{N}\left(1-\alpha_{3}^{j}\right)\right)\left\|x_{n_{i}}-q\right\|^{2}\right) \\
& =\liminf _{i \rightarrow \infty}\left(\prod_{j=1}^{N}\left(1-\alpha_{3}^{j}\right)\left\|x_{n_{i}}-q\right\|^{2}\right. \\
& \left.\quad+\left(1-\prod_{j=1}^{N}\left(1-\alpha_{3}^{j}\right)\right)\left\|x_{n_{i}}-q\right\|^{2}\right) \\
& =\liminf _{i \rightarrow \infty}\left\|x_{n_{i}}-q\right\|^{2} .
\end{aligned}
$$

This is a contradiction. Then, we have $q \in F(S)$. From Lemma 2.9, we have

$$
q \in \bigcap_{i=1}^{N} F\left(T_{i}\right)
$$

From (3.22) and (3.23), we have $q \in \mathfrak{F}$. Hence, $\omega\left(x_{n}\right) \subset \mathfrak{F}$. Therefore, by (3.5) and Lemma 2.8, we have $\left\{x_{n}\right\}$ converges strongly to $P_{\mathfrak{F}} x_{1}$. This completes the proof.

The following result can be obtained from Theorem 3.1. We, therefore, omit the proof.

Corollary 3.2 Let $C$ be a nonempty closed convex subset of a Hilbert space $H$. For every $i=1,2, \ldots, N$, let $A_{i}: C \rightarrow H$ be an $\alpha_{i}$-inverse strongly monotone mapping, and let $T: C \rightarrow$ $C$ be a nonspreading mapping with $\mathfrak{F}=F(T) \cap \bigcap_{i=1}^{N} V I\left(C, A_{i}\right) \neq \emptyset$. For every $i=1,2, \ldots, N$, define the mapping $G_{i}: C \rightarrow C$ by $G_{i} x=P_{C}\left(I-\lambda A_{i}\right) x \forall x \in C$ and $\lambda \in[c, d] \subset\left(0,2 \alpha_{i}\right)$. Let $\left\{x_{n}\right\}$ be a sequence generated by $x_{1} \in C_{1}=C$ and

$$
\left\{\begin{array}{l}
z_{n}=\sum_{i=1}^{N} \delta_{n}^{i} G_{i} x_{n}, \\
y_{n}=\alpha_{n} x_{n}+\beta_{n} T x_{n}+\gamma_{n} z_{n}, \\
C_{n+1}=\left\{z \in C_{n}:\left\|y_{n}-z\right\| \leq\left\|x_{n}-z\right\|\right\}, \\
x_{n+1}=P_{C_{n+1}} x_{1}, \quad \forall n \geq 1,
\end{array}\right.
$$

where $\left\{\alpha_{n}\right\},\left\{\beta_{n}\right\},\left\{\gamma_{n}\right\} \subseteq[0,1], \alpha_{n}+\beta_{n}+\gamma_{n}=1$ and suppose the following conditions hold:

(i) $\lim _{n \rightarrow \infty} \delta_{n}^{i}=\delta^{i} \in(0,1), \quad \forall i=1,2, \ldots, N$ and $\sum_{i=1}^{N} \delta_{n}^{i}=1$;

(ii) $\left\{\alpha_{n}\right\},\left\{\beta_{n}\right\},\left\{\gamma_{n}\right\} \subseteq[a, b] \subset(0,1)$.

Then the sequence $\left\{x_{n}\right\}$ converges strongly to $P_{\mathfrak{F}} x_{1}$.

Corollary 3.3 Let $C$ be a nonempty closed convex subset of a Hilbert space H. Let $A$ : $C \rightarrow H$ be an $\alpha$-inverse strongly monotone mapping, and let $\left\{T_{i}\right\}_{i=1}^{N}$ be a finite family of nonspreading mappings with $\mathfrak{F}=\bigcap_{i=1}^{N} F\left(T_{i}\right) \cap V I(C, A) \neq \emptyset$. Let $\rho_{j}=\left(\alpha_{1}^{j}, \alpha_{2}^{j}, \alpha_{3}^{j}\right) \in I \times I \times I$, $j=1,2,3, \ldots, N$, where $I=[0,1], \alpha_{1}^{j}+\alpha_{2}^{j}+\alpha_{3}^{j}=1, \alpha_{1}^{j}, \alpha_{3}^{j} \in(0,1)$ for all $j=1,2, \ldots, N-1$ and 
$\alpha_{1}^{N} \in(0,1], \alpha_{3}^{N} \in[0,1), \alpha_{2}^{j} \in(0,1)$ for all $j=1,2, \ldots, N$, and let $S$ be the $S$-mapping generated by $T_{1}, T_{2}, \ldots, T_{N}$ and $\rho_{1}, \rho_{2}, \ldots, \rho_{N}$. Let $\left\{x_{n}\right\}$ be a sequence generated by $x_{1} \in C_{1}=C$ and

$$
\left\{\begin{array}{l}
y_{n}=\alpha_{n} x_{n}+\beta_{n} S x_{n}+\gamma_{n} P_{C}(I-\lambda A) x_{n}, \\
C_{n+1}=\left\{z \in C_{n}:\left\|y_{n}-z\right\| \leq\left\|x_{n}-z\right\|\right\} \\
x_{n+1}=P_{C_{n+1}} x_{1}, \quad \forall n \geq 1,
\end{array}\right.
$$

where $\left\{\alpha_{n}\right\},\left\{\beta_{n}\right\},\left\{\gamma_{n}\right\} \subseteq[a, b] \subset(0,1), \alpha_{n}+\beta_{n}+\gamma_{n}=1$ and $\lambda \subseteq[c, d] \subset(0,2 \alpha)$. Then the sequence $\left\{x_{n}\right\}$ converges strongly to $P_{\mathfrak{F}} x_{1}$.

\section{Competing interests}

The authors declare that they have no competing interests.

\section{Acknowledgements}

This research was supported by the Research Administration Division of King Mongkut's Institute of Technology, Ladkrabang.

Received: 25 February 2012 Accepted: 5 October 2012 Published: 24 October 2012

\section{References}

1. Kohsaka, F, Takahashi, W: Fixed point theorems for a class of nonlinear mappings related to maximal monotone operators in Banach spaces. Arch. Math. 91, 166-177 (2008)

2. Chang, SS, Joseph Lee, HW, Chan, CK: A new method for solving equilibrium problem fixed point problem and variational inequality problem with application to optimization. Nonlinear Anal. 70, 3307-3319 (2009)

3. Nadezhkina, N, Takahashi, W: Weak convergence theorem by an extragradient method for nonexpansive mappings and monotone mappings. J. Optim. Theory Appl. 128, 191-201 (2006)

4. Yao, JC, Chadli, O: Pseudomonotone complementarity problems and variational inequalities. In: Crouzeix, JP, Haddjissas, N, Schaible, S (eds.) Handbook of Generalized Convexity and Monotonicity, pp. 501-558. Springer, Netherlands (2005)

5. Yao, Y, Yao, JC: On modified iterative method for nonexpansive mappings and monotone mappings. Appl. Math. Comput. 186(2), 1551-1558 (2007)

6. liduka, H, Takahashi, W: Weak convergence theorem by Cesàro means for nonexpansive mappings and inverse-strongly monotone mappings. J. Nonlinear Convex Anal. 7, 105-113 (2006)

7. Takahashi, W, Takeuchi, Y, Kubota, R: Strong convergence theorems by hybrid methods for families of nonexpansive mappings in Hilbert spaces. J. Math. Anal. Appl. 341, 276-286 (2008)

8. lemoto, S, Takahashi, W: Approximating common fixed points of nonexpansive mappings and nonspreading mappings in a Hilbert space. Nonlinear Anal. 71, 2082-2089 (2009)

9. Takahashi, W: Nonlinear Functional Analysis. Yokohama Publishers, Yokohama (2000)

10. Bruck, RE: Properties of fixed point sets of nonexpansive mappings in Banach spaces. Trans. Am. Math. Soc. 179, 251-262 (1973)

11. Browder, FE: Nonlinear operators and nonlinear equations of evolution in Banach spaces. Proc. Symp. Pure Math. 18 78-81 (1976)

12. Matines-Yanes, C, Xu, HK: Strong convergence of the CQ method for fixed point iteration processes. Nonlinear Anal. 64, 2400-2411(2006)

13. Kangtunyakarn, A, Suantai, S: Hybrid iterative scheme for generalized equilibrium problems and fixed point problems of finite family of nonexpansive mappings. Nonlinear Anal. Hybrid Syst. 3, 296-309 (2009)

doi:10.1186/1687-1812-2012-188

Cite this article as: Kangtunyakarn: Strong convergence of the hybrid method for a finite family of nonspreading mappings and variational inequality problems. Fixed Point Theory and Applications 2012 2012:188. 